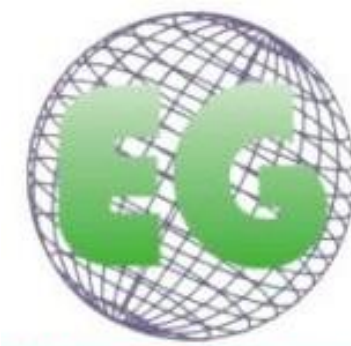

ISSN 1695-6141 $N^{\circ} 48$

Evolución del proceso de afrontamiento y adaptación en adultos hospitalizados que experimentaron un trauma músculo esquelético por accidentes de tránsito

The evolution of coping and adaptation in hospitalised adults who have suffered traffic accident-related musculoskeletal trauma

Ligia Adriana Ávila Martín ${ }^{1}$

Paola Sarmiento ${ }^{2}$

Lucy Muñoz de Rodríguez ${ }^{3}$

${ }^{1}$ Enfermera, Magister en Enfermería. Universidad de la Sabana, Chía, Cundinamarca.Colombia

${ }^{2}$ Profesora de planta tiempo completo, Facultad de Enfermería y Rehabilitación, Universidad de la Sabana, Colombia

${ }^{3}$ Profesora. Facultad de Enfermería, Universidad Nacional de Colombia, Colombia

E-mail: paola.sarmiento1@unisabana.edu.co

http://dx.doi.org/10.6018/eglobal.16.4.255671

Recibido: 04/04/2016

Aceptado: 19/07/2016

\title{
RESUMEN:
}

Objetivo: Explorar la evolución del proceso de afrontamiento y adaptación de 11 casos de adultos que experimentaron un trauma músculo esquelético, por accidentes de tránsito, durante la estancia hospitalaria en la Clínica Universidad de la Sabana en 2015.

Método: Estudio de casos que permitió describir y comparar el proceso de afrontamiento y adaptación en 11 personas. Se aplicó la Escala de medición del proceso de afrontamiento y adaptación versión abreviada en español, al ingreso y al egreso hospitalario. En esta investigación se tuvo en cuenta los principios éticos correspondientes, se obtuvo el consentimiento informado de los participantes y las autorizaciones de la Subcomisión de Investigación de la Facultad de Enfermería y Rehabilitación de la Universidad de la Sabana y de la Clínica donde se llevó a cabo el estudio.

Resultado: Los hallazgos de este estudio muestran para ocho de los once casos una tendencia a mantenerse en nivel alto de afrontamiento o a pasar de nivel medio a alto desde el ingreso hasta el egreso hospitalario.

Conclusión: La mayoría de los casos tuvo un proceso de afrontamiento y adaptación dirigido al problema, donde se encontró interés por resolver dudas relacionadas con la situación antes de actuar, utilización de experiencias pasadas consideradas como útiles, adopción de nuevas habilidades para afrontar las situaciones difíciles y el uso de soluciones alternativas y recursos.

Palabras clave: Enfermería; afrontamiento; adaptación; trauma músculo esquelético; accidentes de tránsito 


\begin{abstract}
:
Objective: Exploring the evolution of coping and adaptation strategies by 11 cases of adults who had suffered traffic accident-related musculoskeletal trauma during their stay in hospital at Clínica Universidad de la Sabana during 2015.

Methods: Studying the aforementioned cases enabled describing and comparing the 11 patients' coping and adaptation strategies. The abridged Spanish version of Roy's Coping Adaptation Processing Scale (CAPS) was used for measuring such strategies; it was used on patient admission to and discharge from hospital. This research took the relevant ethical principles into account; the patients gave their written informed consent and the Universidad de la Sabana and its hospital where the study was carried out authorised the study (i.e. the Faculty of Nursing and Rehabilitation's Research Subcommittee).

Results: This study's findings showed that eight of the eleven cases maintained a high coping ability, or improved from moderate (on admission to hospital) to high level ability (on discharge).

Conclusion: Problem-centred coping and adaptation strategies were involved in most cases. Patients approached this through resolving situation-related questions before acting, using past experiences considered useful, adopting new skills to cope with difficult situations and using alternative solutions and resources.
\end{abstract}

Palavras-chave: Nursing; coping; adaptation; musculoskeletal trauma; traffic accidents

\title{
INTRODUCCIÓN
}

El trauma músculo esquelético que experimentan las personas, se produce cuando el cuerpo recibe un impacto que altera las funciones propias de los órganos que resultan afectados $^{(1)}$, puede ser causado por una variedad de mecanismos, pero cualquiera que sea su causa, el punto en común es el daño celular, en tejidos y órganos a causa de la transmisión externa de fuerzas al cuerpo ${ }^{(2)}$. Estos traumas constituyen para las personas un importante problema de salud durante toda la vida. Actualmente proyectados a convertirse en la cuarta causa de discapacidad ajustada a los años de vida para el 2030 a nivel mundial según Mathers et al. ${ }^{(2)}$, se clasifican dentro de las diez primeras causas de muerte a nivel global $^{(2)}$ y se ha reportado que una de sus principales causas de aparición son los accidentes automovilísticos.

El impacto social de esta nueva pandemia es tan grave, que produce aproximadamente 3,5 millones de muertes y alrededor de 50 millones de lesionados anualmente en el mundo, con daños que pueden ir desde lesiones leves hasta secuelas severas con discapacidad importante ${ }^{(3)}$.

En Colombia, según Quintero(1) ${ }^{(1)}$ las elevadas cifras de morbilidad y mortalidad por traumatismos, ocasionan anualmente hasta el $41 \%$ de los años de vida saludables perdidos por discapacidades y afectan en especial al grupo laboral de personas económicamente activas comprendido entre los 15 y 45 años de edad. Como lo informa también el Instituto Nacional de Medicina Legal y Ciencias Forenses para el año 2014 que reporta 50.574 casos atendidos por accidentes de transporte durante el año; con un $87.3 \%$ de casos que corresponden a lesiones no fatales, o sea 44.172 personas; durante el año 2014 el aumento de heridos por accidentes de transporte fue de 5,62\% comparado con el 2013, siendo el hombre el más afectado en accidentes de transporte tanto en lesiones fatales como no fatales; con respecto a la edad, el $65.06 \%$ de las personas con lesiones no fatales están entre 15 y 44 años, predominante en el grupo etario de 20 a 24 años de edad ${ }^{(4)}$.

Por otro lado, como menciona Martín ${ }^{(5)}$, estas situaciones traumáticas en adultos se han relacionado con el impacto psicológico y con la aparición de trastornos psiquiátricos, ya que corresponden a situaciones que van acompañadas de una mezcla de consecuencias psicológicas, médicas y legales. La persona 
politraumatizada sufre intranquilidad y confusión por las circunstancias, el medio desconocido y el temor hacia la muerte, la mutilación, la inmovilización y otras alteraciones de su identidad e integridad corporal, como efectos que pueden sobrevenir del trauma ${ }^{(1)}$

Por ejemplo, Cáceres ${ }^{(6)}$ reportó que después de un evento traumático la persona se ve enfrentada a una serie de pérdidas en diferentes aspectos de su vida, pasando por una etapa de desconcierto e incredulidad, una toma de conciencia progresiva, que se acompaña de sentimientos de tristeza, culpa, desesperanza, trastornos del sueño y de la alimentación; produciéndose alteraciones en su estado emocional que pueden afectar la forma como la persona afronta el proceso de rehabilitación y como se adapta a sus nuevas condiciones de vida; requiriendo de la persona el desarrollo de su capacidad de afrontamiento.

A su vez, Zavala ${ }^{(7)}$ señala que ante un estímulo estresante proveniente del medio, la persona realiza una evaluación y proporciona estrategias de afrontamiento a las que se suman expresiones emocionales, generando como consecuencia una serie de respuestas. Por lo tanto, para saber si un evento es vivido como estresante, es necesario conocer la interpretación que la persona hace de él (Lazarus, 1991); mientras que para una persona un evento traumático puede constituir un obstáculo, para otro puede suponer una oportunidad para tomar conciencia y reestructurar la forma de entender el mundo ${ }^{(8)}$.

Es así, como algunas personas suelen resistir con insospechada fortaleza los embates de la vida, e incluso ante sucesos extremos hay un elevado porcentaje de personas que muestra una gran resistencia y que sale psicológicamente indemne o con daños mínimos del evento ${ }^{(8)}$. De hecho, las teorías que defienden la posibilidad de crecimiento o aprendizaje postraumático, adoptan la premisa de que la adversidad puede, a veces, perder parte de su severidad gracias a procesos cognitivos de adaptación, consiguiendo no sólo restaurar las visiones adaptativas de uno mismo, los demás y del mundo, sino también fomentar la convicción de que uno es mejor de lo que era antes del suceso ${ }^{(8)}$.

Aunque los datos apuntan a que aproximadamente un $85 \%$ de las personas afectadas por una experiencia traumática siguen un proceso natural de recuperación y no desarrollan ningún trastorno(8), para muchas personas que experimentaron un accidente de tránsito, sigue habiendo gran dificultad para completar la adaptación, dado el continuo desafío psicológico, social, físico, legal y financiero ${ }^{(9)}$.

Este tema ha sido abordado por disciplinas como Psicología, Medicina, Psiquiatría y Enfermería, que se han enfocado en reportar las consecuencias que implica para las personas experimentar este tipo de eventos, haciendo énfasis en las consecuencias psicológicas, el riesgo de desarrollar trastornos psiquiátricos ${ }^{(5,10-12)}$, la calidad de vida después del trauma ${ }^{(13,14)}$, el significado de la experiencia de la persona politraumatizada ${ }^{(1)}$, sus percepciones acerca de esta vivencia, acerca del cuidado de Enfermería recibido en estas circunstancias ${ }^{(9,15,16)}$ y las intervenciones educativas creadas para este grupo de pacientes ${ }^{(6,17,18,)}$. Además, investigaciones de origen internacional de tipo cualitativo y cuantitativo han reportado estudios sobre el afrontamiento en estas personas ${ }^{(19-25)}$ donde destacan la importancia que tiene identificar la capacidad de afrontamiento y adaptación para el diseño de intervenciones tempranas que puedan disminuir el riesgo de desarrollar alteraciones relacionadas con el trauma, ya que identificaron que los estilos de afrontamiento se 
relacionan con resultados psicológicos variados y cambios en la funcionalidad y calidad de vida $\left.{ }^{(26}\right)$.

Sin embargo, en la búsqueda preliminar realizada no se hallaron estudios investigativos a nivel nacional asociados a este fenómeno de estudio, su evolución en el tiempo, las estrategias que usaron para afrontar la enfermedad y como llevan a cabo la adaptación a sus nuevos estilos de vida, por lo que resulta útil explorar el proceso de afrontamiento y adaptación de adultos que experimentaron un trauma músculo esquelético por accidentes de tránsito a través del análisis de estudios de caso, para conocer la profundidad este fenómeno poco estudiado en nuestro país desde la fundamentación teórica de Enfermería.

Por lo que surge la pregunta de ¿Cómo es la evolución del proceso de afrontamiento y adaptación de 11 casos de adultos que experimentaron un trauma músculo esquelético por accidentes de tránsito durante la estancia hospitalaria en la Clínica Universidad de la Sabana en 2015?

Con el objetivo de explorar la evolución del proceso de afrontamiento y adaptación de 11 casos de adultos que experimentaron un trauma músculo esquelético, por accidentes de tránsito durante la estancia hospitalaria en la Clínica Universidad de la Sabana, a través de la descripción del proceso de afrontamiento y adaptación al inicio de la estancia hospitalaria, al egreso de la Clínica Universidad de la Sabana y comparar los resultados.

\section{MATERIALES Y MÉTODOS}

El diseño de esta investigación corresponde a estudio de casos, ya que permite describir y comparar el proceso de afrontamiento y adaptación en 11 casos de personas, con el fin de profundizar en este fenómeno. También se considera de tipo descriptivo comparativo, porque contrasta la capacidad de afrontamiento y adaptación de adultos que experimentaron un trauma músculo esquelético como resultado de accidentes de tránsito, en dos momentos distintos durante un período de tiempo determinado, con la finalidad de examinar sus variaciones en el tiempo ${ }^{(27)}$.

Corresponde a una muestra no probabilística de 11 casos de personas que experimentaron trauma musculo esquelético como resultado de accidentes de tránsito, que se encontraban en hospitalización de la Clínica Universidad de la Sabana de enero a diciembre de 2015, que cumplieron con los criterios de inclusión y a quienes se logró aplicar la Escala de Medición del proceso de afrontamiento y adaptación al ingreso y al egreso del servicio de Hospitalización de la Clínica.

Criterios de inclusión: Adultos de ambos sexos mayores de 18 años, con Glasgow $15 / 15$, con las condiciones físicas y mentales para responder a la escala que ingresaron al servicio de hospitalización de la Clínica Universidad de la Sabana por trauma músculo esquelético por accidente de tránsito.

Criterios de exclusión: Lesiones traumáticas del cerebro, estado hemodinámico inestable, antecedente de enfermedad médica grave e historia de trastornos psiquiátricos.

En este trabajo se utilizó la Escala de Medición del Proceso de Afrontamiento y Adaptación de Callista Roy (Coping Adaptation Processing Scale - CAPS) versión 
final abreviada y modificada compuesta por 33 ítems, que reportó un índice de validez de contenido global para esta versión de 0.83, consistencia interna a través del alfa de Cronbach reportó una confiabilidad de 0,70 y una validez facial del $95 \%{ }^{(28)}$. Esta escala en su versión original está integrada por 47 ítems, cada ítem corresponde a una frase corta acerca de cómo una persona responde cuando experimenta una crisis o un evento difícil. Se hizo uso de la última versión, ya que dentro de las limitaciones encontradas por Gutiérrez et al., ${ }^{(29)}$ la escala original era compleja en su constructo y de difícil aplicación por su semántica y extensión.

El proceso de recolección de la información se desarrolló así:

1. Selección de los participantes previa verificación de criterios de inclusión y exclusión.

2. Diligenciamiento de consentimiento informado.

3. Aplicación de la CAPS a las personas que autorizaron y consintieron su participación en la investigación, se aplicó de forma administrada al ingreso y al egreso hospitalario en tiempo fuera del horario de visitas, garantizando un ambiente de tranquilidad, privacidad, evitando al máximo interrupciones como procedimientos médicos o de enfermería simultáneos.

4. Registro de los resultados de la aplicación de la escala en formulario de google drive.

Procesamiento de la información:

1. Se usó la estadística descriptiva básica para el análisis de los datos sociodemográficos en los 11 casos.

2. Se compararon los resultados de la primera con la segunda medición según la CAPS.

3. Se realizó un cuadro comparativo de los 11 casos, para facilitar su análisis.

4. Se realizó análisis de cada caso comparando los resultados según el nivel de afrontamiento (alto, medio y bajo) de acuerdo a la puntuación total establecida en la CAPS.

\section{Consideraciones éticas}

La presente investigación tuvo en cuenta los principios éticos generales de la investigación en seres humanos, de acuerdo con la Council for International Organizations of Medical Sciences (ClOMS) ${ }^{(30)}$.

También se tuvo en cuenta los principios que plantea la declaración de Helsinki acerca del deber de proteger la vida, la salud, la integridad, el derecho a la autodeterminación, la intimidad y la dignidad del ser humano (Declaración de Helsinki de la Asociación Médica Mundial, 2002)(31).

Se consideraron los lineamientos éticos del Código de Núremberg (1947) y las pautas del consentimiento informado establecidas por el informe de Belmont, con la información suficiente sobre la naturaleza, el propósito, el método, los medios, la duración, los beneficios, los riesgos y las alternativas existentes de forma clara, completa y comprensible ${ }^{(31)}$. 
Se tuvo en cuenta las políticas y normas institucionales donde se desarrolló la investigación y se contó con la autorización del Comité de ética de la Universidad de la Sabana; el manejo de la información generado por la implementación de la Escala se trató de forma confidencial.

La metodología del presente trabajo investigativo no establece riesgos para los participantes en conformidad con la resolución $008430^{(32)}$.

\section{RESULTADOS}

\section{Descripción socio demográfica de los casos}

Clasificación según la edad: El rango de edad de los participantes en el estudio estuvo entre los 18 y 50 años, con mayor número de personas entre los 18 y 24 años y entre los 35 a 40 años. Estas cifras se acercan a la estadística nacional, donde con respecto a la edad, el $65.06 \%$ de las personas con lesiones no fatales están entre los 15 y 44 años, predominante en el grupo etario de 20 a 24 años de edad, según el Instituto Nacional de Medicina Legal y Ciencias Forenses para el año $2014^{(4)}$.

Tabla 1. Clasificación según el sexo

\begin{tabular}{|l|l|}
\hline Sexo & Frecuencia \\
\hline Masculino & 9 \\
\hline Femenino & 2 \\
\hline Total & 11 \\
\hline
\end{tabular}

Fuente: Datos recolectados de la aplicación de la Escala de medición del proceso de afrontamiento y adaptación de Callista Roy en la Clínica Universidad de la Sabana, 2015.

Se observa más hombres que mujeres afectados por accidentes de tránsito, dato similar a lo reportado por el Instituto Nacional de Medicina Legal y Ciencias Forenses para el año 2014 y en investigaciones Nacionales e Internacionales sobre el fenómeno de estudio $(10,14,17,18)$

Clasificación según escolaridad: Más de la mitad de los casos corresponde a personas con nivel educativo de bachillerato completo, lo cual coincide con hallazgos del Instituto Nacional de Ciencias Forenses para el $2014^{(4)}$, donde se especifica que las personas heridas por accidentes de transporte, con nivel de escolaridad de básica secundaria representaron un acumulado de $79,92 \%$ de los casos y con lo reportado en el análisis de datos del estudio realizado por Mi-Ling Wong et al., ${ }^{(17)}$ donde el $52 \%$ de los pacientes con traumas musculo esqueléticos que hicieron parte del estudio tenían educación secundaria.

Clasificación según estado civil: El estado civil soltero ocupa el primer lugar en este estudio, así como en las estadísticas nacionales proporcionadas por el Instituto Nacional de Medicina Legal y Ciencias Forenses para el 2014, con tasas válidas cercanas al $41 \%$ en personas con lesiones no fatales ${ }^{(4)}$.

Clasificación según el tipo de lesión: 5 casos correspondieron a lesiones de tipo fractura de tibia y peroné; que coincide con el estudio de Fitzharris et al. 2006, donde el $46.8 \%$ corresponde a lesiones de las extremidades inferiores en pacientes involucrados en accidentes de tránsito ${ }^{(33)}$ (Tabla 2) 
Tabla 2.

\begin{tabular}{|l|c|}
\hline Tipo de lesión & $\begin{array}{l}\text { Frecuenci } \\
\text { a }\end{array}$ \\
\hline $\begin{array}{l}\text { Herida compleja de la mano } \\
\text { y la pierna }\end{array}$ & 1 \\
\hline Trauma cerrado de tórax & 1 \\
\hline Fractura de tibia y peroné & 5 \\
\hline Fractura de fémur derecho & 2 \\
\hline Politraumatismo & 1 \\
\hline Fractura de cadera & 1 \\
\hline Total & 11 \\
\hline
\end{tabular}

Fuente: Datos recolectados de la aplicación de la Escala de medición del proceso de afrontamiento y adaptación de Callista Roy en la Clínica Universidad de la Sabana, 2015.

Clasificación de acuerdo a la condición durante el accidente: De los 11 casos, 6 tuvieron la condición de conductores durante el accidente de tránsito, datos que también fueron reportados por el Instituto Nacional de Medicina Legal y Ciencias Forenses para el 2014 , donde el $67 \%$ de las víctimas tenía condición de conductores durante el accidente; también coincide con los hallazgos encontrados en el estudio de Ching-Hui Wang et al., en 2005, donde el $89 \%$ de los participantes correspondían a conductores ${ }^{(10)}$.

Clasificación de acuerdo al tiempo de estancia hospitalaria: De los 11 casos, en 8 se reportó de 4 a 7 días de estancia hospitalaria, que resulta inferior a lo reportado por O'Donnell ${ }^{(18)}$ con un promedio de 8,2 días de estancia hospitalaria para sus participantes. Sin embargo, puede estar relacionado con el tipo de lesión y con la iniciativa de la Unidad Estratégica de Hospitalización de la Clínica para el 2014, donde se redujo el promedio de días de estancia hospitalaria a 3,4 días; basado en las necesidades de los pacientes y con el objetivo de generar egresos oportunos, seguros y aumento del giro cama ${ }^{(34)}$.

Descripción de casos según Escala de medición del proceso de afrontamiento y adaptación (CAPS)

Al finalizar la descripción de cada uno de los casos se totaliza la puntuación, que para esta escala oscila entre una mínima de 33 y máxima de 132, con los siguientes resultados: afrontamiento bajo: 33-89 afrontamiento medio: 90-103 afrontamiento alto: $104-132^{(28)}$. 
Tabla 3. Comparación de los 11 casos de estudio. Esta tabla consolida de manera comparativa los resultados de la CAPS para los 11 casos:

\begin{tabular}{|c|c|c|c|c|}
\hline DATOS SOCIODEMOGRÁFICOS & $\begin{array}{l}\text { AFRONTA } \\
\text { MIENTO } 1\end{array}$ & $\begin{array}{l}\text { PUN } \\
\text { TA } \\
\text { JE }\end{array}$ & $\begin{array}{l}\text { AFRON } \\
\text { TA } \\
\text { MIENTO } \\
2\end{array}$ & $\begin{array}{l}\text { PUN } \\
\text { TAJE }\end{array}$ \\
\hline $\begin{array}{l}\text { CASO N 1. Hombre de } 34 \text { años, conductor, con estrato } \\
\text { socioeconómico } 2 \text {. Escolaridad: bachillerato completo, } \\
\text { casado, con múltiples heridas en mano y herida compleja } \\
\text { en la pierna. con } 8 \text { días de hospitalización. }\end{array}$ & MEDIO & 103 & ALTO & 109 \\
\hline $\begin{array}{l}\text { CASO N 2. Mujer de } 39 \text { años, pasajera, con estrato } \\
\text { socioeconómico número } 2 \text {. Escolaridad: bachillerato } \\
\text { incompleto. Estado civil: Unión libre, con trauma cerrado de } \\
\text { tórax y tiempo de hospitalización de } 5 \text { días. }\end{array}$ & MEDIO & 94 & ALTO & 104 \\
\hline $\begin{array}{l}\text { CASO N 3. Hombre de } 45 \text { años, conductor, con estrato } \\
\text { socioeconómico } 2 \text {. Escolaridad: bachillerato completo, en } \\
\text { unión libre, con fractura de tibia y peroné y } 6 \text { días de } \\
\text { hospitalización. }\end{array}$ & MEDIO & 98 & ALTO & 104 \\
\hline $\begin{array}{l}\text { CASO N 4. Hombre de } 42 \text { años, pasajero, con estrato } \\
\text { socioeconómico } 2 \text {. Escolaridad: bachillerato completo. } \\
\text { Estado civil: Casado, con fractura de fémur derecho - } \\
\text { trauma cerrado de abdomen y } 31 \text { días de hospitalización. }\end{array}$ & MEDIO & 102 & ALTO & 110 \\
\hline $\begin{array}{l}\text { CASO N 5. Mujer de } 48 \text { años, peatón, con estrato } \\
\text { socioeconómico 1. Escolaridad: primaria completa, } \\
\text { separada, con trauma cerrado de tórax, fractura de tibia y } \\
\text { peroné y trauma de cadera y } 5 \text { días de hospitalización. }\end{array}$ & MEDIO & 100 & ALTO & 105 \\
\hline $\begin{array}{l}\text { CASO N 6. Hombre de } 40 \text { años, conductor, con estrato } \\
\text { socioeconómico 1. Escolaridad: bachillerato incompleto, } \\
\text { soltero, con fractura de platillos tibiales izquierdos y } 4 \text { días } \\
\text { de estancia hospitalaria. }\end{array}$ & ALTO & 109 & MEDIO & 98 \\
\hline $\begin{array}{l}\text { CASO N 7. Hombre de } 24 \text { años, conductor, con estrato } \\
\text { socioeconómico } 3 \text {. Escolaridad: bachillerato completo, } \\
\text { soltero, con fractura abierta de tibia y peroné y } 48 \text { días de } \\
\text { hospitalización. }\end{array}$ & ALTO & 111 & ALTO & 108 \\
\hline $\begin{array}{l}\text { CASO N 8. Hombre de } 33 \text { años, conductor, con estrato } \\
\text { socioeconómico } 3 \text {. Escolaridad: bachillerato completo, en } \\
\text { unión libre, con fractura de fémur derecho y } 5 \text { días de } \\
\text { hospitalización. }\end{array}$ & ALTO & 105 & ALTO & 106 \\
\hline $\begin{array}{l}\text { CASO N 9. Hombre de } 23 \text { años, peatón, con estrato } \\
\text { socioeconómico } 2 \text {. Escolaridad: universitario, soltero, con } \\
\text { cuadro de politraumatismo y } 10 \text { días de hospitalización. }\end{array}$ & MEDIO & 97 & ALTO & 104 \\
\hline $\begin{array}{l}\text { CASO N 10. Hombre de } 35 \text { años, peatón, con estrato } \\
\text { socioeconómico 3. Escolaridad: primaria incompleta, } \\
\text { soltero, con cuadro de fractura de tibia izquierda y } 4 \text { días } \\
\text { de hospitalización. }\end{array}$ & BAJO & 88 & MEDIO & 93 \\
\hline $\begin{array}{l}\text { CASO N 11. Hombre de } 21 \text { años, conductor, con estrato } \\
\text { socioeconómico } 3 \text {. Escolaridad: bachillerato completo, } \\
\text { soltero, con fractura de cadera y } 7 \text { días de hospitalización. }\end{array}$ & ALTO & 107 & MEDIO & 93 \\
\hline
\end{tabular}

De los 11 casos reportados, 6 casos (caso 1,2,3,4,5 y 9) obtuvieron nivel de afrontamiento y adaptación medio en la primera medición al momento del ingreso hospitalario y nivel alto en la segunda medición, al momento del egreso; de estos 6 casos, se encuentran 4 hombres, 2 mujeres, con edades entre los 34 y 48 años de edad, predominantemente; a excepción de un adulto joven de 23 años, el estrato 
socioeconómico que prepondera es el 2 y un adulto con estrato socioeconómico 1. La condición durante el accidente, el tipo de lesión, la escolaridad y el tiempo de hospitalización se encuentra variada en los 6 casos.

En los 6 casos anteriores, se observó un afrontamiento activo, dirigido al problema, donde a los individuos les interesa buscar información sobre qué hacer para eliminar definitivamente el problema haciendo uso de soluciones alternativas y recursos ${ }^{(35)}$; este tipo de afrontamiento está caracterizado por mirar la situación actual en forma positiva, sintiéndose bien de saber que maneja la situación lo mejor que puede, llevando a la persona a utilizar la re-evaluación para modificar o tolerar la situación, favoreciendo su adaptación ${ }^{(36)}$. El estilo de afrontamiento activo está caracterizado por el dominio conductual manifestado por búsqueda y recopilación de información, estar atento a cualquier cosa relacionada con la situación desde el principio, la identificación de posibles soluciones, el desarrollo de un plan para el logro de los objetivos y la proposición de soluciones nuevas para resolverlo ${ }^{(36)}$. Este proceso cognitivo de tomar acción directa, planear el futuro, manejar el uso sistemático de recursos y experiencias pueden afectar las respuestas adaptativas en alta autoestima, transición de rol y actitudes saludables ${ }^{(37)}$.

Además, tres de los seis casos ( $\operatorname{casos} 1,2$ y 9) utilizan las soluciones que le han funcionado a otros para resolver sus situaciones difíciles o problemas, que resulta en una estrategia de afrontamiento activo, dirigido a la solución del problema, caracterizada por comportamientos que implican el aprendizaje de experiencias pasadas, el recordar soluciones y cosas que sirvieron y ayudaron en situaciones anteriores y el aprender de las soluciones que le funcionaron a otros para resolver los propios problemas $^{(36)}$

Siguiendo con el análisis, de los 11 casos, 2 obtuvieron nivel de afrontamiento y adaptación alto en la primera medición al momento del ingreso y nivel medio en la segunda medición, al egreso (casos 6 y 11); de estos 2 casos con nivel de afrontamiento alto y posteriormente medio, se encuentran dos hombres de 40 y 21 años, que comparten como datos similares el ser conductores y solteros, los demás datos sociodemográficos son variables entre los dos adultos.

A pesar de que la capacidad de afrontamiento y adaptación de estos dos casos no mejoró en el tiempo, sino que por el contrario, disminuyó, se observa un tipo de afrontamiento dirigido al problema, manifestado por comportamientos de búsqueda y recopilación de información, el desarrollo de un plan para el logro de los objetivos y la proposición de soluciones nuevas. Sin embargo es posible deducir de acuerdo a las respuestas con tendencia negativa, que los casos 6 y 11 también pudieron haber adoptado algunas conductas de escape-evitación, minimización de la amenaza (Buckelew et al., 1990, 1991) $^{(23)}$, dificultad para relacionar las situaciones difíciles con experiencias pasadas y con planes futuros y para usar las soluciones que le han funcionado a otros para resolver sus situaciones difíciles, lo que dificulta el desarrollo de capacidades para la solución del problema, ya que el subsistema cognitivo le permite a la persona crear nuevos conocimientos, basados en recuerdos, en la nueva experiencia $^{(38)}$ y en las soluciones que le funcionaron a otros para resolver los problemas.

Los casos 6 y 11, al inicio de la aplicación de la escala, respondieron en positivo a la pregunta: veo las situaciones difíciles o problemas como una oportunidad o un reto, para después pasar a nunca o casi nunca ver las situaciones difíciles como una 
oportunidad o un reto, esto puede resultar en una conducta poco adaptativa, en cuanto a que aquellas personas para las que los acontecimientos significan un reto probablemente tengan muchas más ventajas en cuanto a funcionamiento y salud que aquellas que fácilmente se sienten amenazadas ${ }^{(39)}$.

Mientras tanto, los casos 7 y 8 , que tuvieron nivel de afrontamiento y adaptación alto en las dos mediciones durante la estancia hospitalaria, son hombres de 24 y 33 años, conductores, con estrato socioeconómico 3 y bachillerato completo y con variaciones de un caso a otro en datos sociodemográficos como el estado civil, tipo de lesión y tiempo de hospitalización. Se observó un afrontamiento activo, donde los individuos hacen uso de soluciones alternativas y recursos para resolver el problema ${ }^{(35)}$, con uso de la planeación, análisis y aceptación de la realidad del evento estresante tratando de cambiarlo para obtener efectos positivos ${ }^{(40)}$; este estilo de afrontamiento está caracterizado por estar atento a cualquier cosa relacionada con la situación desde el principio y a tener disposición al cambio para salir de problemas. Este proceso cognitivo de tomar acción directa, planear el futuro, manejar el uso sistemático de recursos y experiencias pueden afectar las respuestas adaptativas en alta autoestima, transición de rol, sensación y actitudes saludables ${ }^{(37)}$.

Por último, el caso número 10, se analiza solo, por ser el único con nivel de afrontamiento bajo al inicio de la aplicación de la escala y nivel medio al egreso hospitalario, corresponde a un hombre de 35 años, peatón, con estrato socioeconómico 3, escolaridad primaria incompleta, soltero, con cuadro de fractura de tibia izquierda y 4 días de hospitalización.

En este caso se observó una tendencia positiva en la mayoría de las respuestas a las preguntas de la escala para su segunda medición, sin embargo, también fue posible encontrar un estilo de afrontamiento evasivo, considerado como menos exitoso; se halla la pretensión de negar la situación, ignorar la realidad del evento crítico o desentenderse de la realidad vivida ${ }^{(41)}$, se encontró una estrategia y estilo de afrontamiento pasivo, caracterizado por el dominio conductual, manifestado por inhabilidad para manejar problemas complejos, manejo de situaciones bajo estrés, análisis rápido de la situación, uso de la planeación para enfrentar la situación y pobre disposición al cambio para salir de problemas.

\section{DISCUSION}

Los hallazgos de este estudio muestran para ocho de los once casos una tendencia a mantenerse en nivel alto de afrontamiento o una tendencia a pasar de nivel medio a alto de capacidad de afrontamiento y adaptación desde el ingreso hasta el egreso hospitalario; lo cual es consistente con la literatura encontrada, que sugiere que el ser humano tiene una gran capacidad para adaptarse y encontrar sentido a las experiencias traumáticas más difíciles e incluso hay un elevado porcentaje de personas que muestra una gran resistencia y que sale psicológicamente indemne o con daños mínimos del evento estresante. Desde modelos más optimistas, se entiende que la persona es activa y fuerte, con una capacidad natural de resistir y rehacerse a pesar de las adversidades ${ }^{(8)}$. Inclusive, las teorías que defienden la posibilidad de crecimiento o aprendizaje postraumático adoptan la premisa de que la adversidad puede, a veces, perder parte de su severidad gracias a procesos cognitivos de adaptación, consiguiendo no sólo restaurar las visiones adaptativas de uno mismo, los demás y el mundo, que en un principio podían haberse distorsionado, sino también fomentar la convicción de que uno es mejor de lo que era antes del 
suceso $^{(8)}$. Como recuerda Denholm ${ }^{(19)}$ la experiencia de haber sufrido un traumatismo generó efectos tanto positivos como negativos; sin embargo el hecho de pasar por situaciones difíciles hace que se desarrollen pensamientos y habilidades fortalecedoras.

Además, los resultados hallados en este trabajo de investigación son similares a los encontrados en el estudio de Flórez ${ }^{(42)}$ acerca del nivel de afrontamiento y adaptación en pacientes egresados de la Unidad de Cuidados Intensivos (UCI), algunos de éstos con politraumatismo, donde el $63,2 \%$ de los participantes se ubicó en el nivel de alta capacidad, el $19,9 \%$ en la mediana, y la baja capacidad de afrontamiento y adaptación reportó $1,2 \%$, con hallazgos que señalan altacapacidad de afrontamiento y adaptación en los pacientes egresados de $\mathrm{UCl}$ ante los estímulos focales, contextuales y residuales propios de la hospitalización, reflejando un estilo de afrontamiento centrado en estrategias cognitivas que buscan encontrar significado al suceso y solucionar directamente la situación.

De acuerdo a las respuestas analizadas, en 8 de los 11 casos (casos 1,2,3,4,5,7,8 y 9), se encontró una tendencia positiva en su proceso de afrontamiento en el tiempo, mantuvieron un afrontamiento activo, dirigido al problema, caracterizado por mirar la situación actual en forma positiva y utilizando la re-evaluación para modificar o tolerar la situación; estas características se comparten con el reporte del estudio cualitativo de Gustafsson ${ }^{(24)}$ sobre las estrategias de afrontamiento en pacientes con lesiones traumáticas de la mano, en donde se encontraron estrategias como confiar en la capacidad personal, aceptar la situación tal como es y tratar de hacer lo mejor, volviendo la experiencia negativa en algo positivo. Mantener el control y la solución de problemas prácticos por sí mismos.

También se encontraron similitudes con el análisis cualitativo de Tan et al..$^{(43)}$, donde los pacientes que habían experimentado accidentes de tránsito en vehículo de motor usaron varias estrategias de afrontamiento para sobrevivir al trauma. Algunos participantes tenían el apoyo de su familia y amigos, otros las creencias espirituales y a otros la experiencia pasada del trauma les ayudó a través del viaje de recuperación, concluyendo que existe una relación entre experiencias previas de vida y la capacidad de afrontamiento de la víctima con la experiencia de trauma posterior.

Cabe señalar, que 7 de los 11 casos ( $\operatorname{casos} 1,2,3,4,5,9$ y 10) presentaron variaciones con respecto al tiempo, con tendencia a mejorar su capacidad de afrontamiento y adaptación. Esto se puede relacionar con la literatura que apoya la idea del afrontamiento como un proceso dinámico, que se va modificando para responder a las necesidades cambiantes de la persona ${ }^{(23)}$, complejo, individualizado y no lineal ${ }^{(44)}$, como reportó un estudio en personas con trastornos por latigazo cervical, donde en general, los pacientes mostraron mayor uso de estrategias de afrontamiento con el paso del tiempo ${ }^{(25)}$; también ratifica la literatura propia del trauma, que está de acuerdo en que el período inmediatamente después del trauma es fundamental y la mayoría del afrontamiento ocurre dentro de las primeras semanas y meses después del evento traumático (Brewin, Andrews, Rose, and Kirk 1999; Shalev 2002) ${ }^{(21)}$.

Siguiendo con la discusión, 1 de los 11 casos reportó nivel bajo de afrontamiento y adaptación al inicio de la estancia hospitalaria y aumentó a nivel medio en la segunda medición, en este caso fue posible encontrar un estilo de afrontamiento evasivo, estas formas pasivas consisten en la ausencia de afrontamiento o conductas de evasión y negación, consideradas como menos exitosas; lo cual puede resultar 
contraproducente como una forma individual de no hacer ningún intento de alterar el medio amenazante o sus respuestas al ambiente y a largo plazo puede hacer que las víctimas no puedan traducir la amenaza en algo que es controlable (Bryant \& Harvey, 1995; Rachman, 1990) ${ }^{(45)}$.

Finalmente, los resultados generales de este trabajo son similares a lo encontrado en investigaciones como la de Rueda y Aguado $^{(23)}$ que reportan un afrontamiento más caracterizado por estrategias activas, de resolución de problemas, mayor aceptación y reestructuración cognitiva en individuos con lesiones de la medula espinal, que por estrategias de corte emotivo. Esto también está reportado en el estudio de Hepp et al. ${ }^{(44)}$, donde mencionan al afrontamiento activo, centrado en el problema, como la estrategia de afrontamiento predominante poco después de un accidente.

\section{LIMITACIONES}

El tipo de diseño de estudio de casos, impide generalizar los resultados a la amplia población de adultos con traumas musculo esqueléticos, por lo que sería recomendable un tamaño de muestra más grande que permita obtener resultados estadísticamente significativos. Otra limitación fue el promedio de estancia hospitalaria de los participantes en la Institución donde se desarrolló el presente estudio, pues dificultó la medición de la evolución del proceso de afrontamiento y adaptación en un tiempo superior a 4 días.

\section{CONCLUSIONES}

1. Este estudio permitió explorar la evolución del proceso de afrontamiento y adaptación de 11 casos de adultos con trauma musculo esquelético por accidentes de tránsito, donde se evidenció que más de la mitad de los casos pasó de nivel medio a alto de afrontamiento y adaptación durante la estancia hospitalaria.

2. La mayoría de los casos tuvo un proceso de afrontamiento y adaptación dirigido al problema, un tipo de afrontamiento activo.

3. El tiempo resulta ser una variable a tener en cuenta en el proceso de afrontamiento y adaptación. En esta investigación se encontró que con el tiempo mejoró el proceso de afrontamiento de 9 de los 11 casos estudiados, lo que muestra como una persona puede modificar sus estrategias a través del tiempo.

4. El afrontamiento constituye una variable que permite entender como los individuos le hacen frente a sus dificultades y cómo este proceso se da de forma natural.

5. A pesar que algunos de los casos compartían características sociodemográficas semejantes, su proceso de afrontamiento y adaptación es único, lo que implica que dos personas pueden tener la misma experiencia traumática y mostrar diversas reacciones y formas de afrontamiento.

6. En la mayoría de los casos se evidenció un proceso de afrontamiento adecuado, que recuerda que el ser humano posee capacidad para adaptarse y encontrar sentido a las experiencias traumáticas vividas, convirtiéndose en una oportunidad para aprender de la experiencia, mantener el equilibrio, proyectarse al futuro, creer 
que se puede influir en lo que sucede a su alrededor y que se puede aprender de las experiencias tanto positivas como negativas, logrando hacer que se desarrollen pensamientos y habilidades fortalecedoras que conduzcan a la adaptación.

Surgieron las siguientes hipótesis del trabajo de investigación:

¿El dolor y la alteración de la movilidad influyen en el proceso de afrontamiento y adaptación de adultos con traumas musculo esqueléticos por accidente de tránsito? ¿Puede la intervención de Enfermería mejorar el proceso de afrontamiento y adaptación en el tiempo?

¿Qué intervenciones de Enfermería podrían mejorar el proceso de afrontamiento y adaptación de adultos con traumas músculo esquelético por accidente de tránsito?

\section{RECOMENDACIONES}

- Motivar a los Enfermeros a que inicien el proceso de identificación del nivel de afrontamiento y adaptación con los adultos que tienen a su cuidado, a través de la empatía, la relación de confianza con ellos, fomento de la expresión de sentimientos, evaluación de las situaciones difíciles anteriormente experimentadas y el uso de estrategias útiles que pueden servir de insumo para resolver o minimizar el impacto de las situaciones nuevas, con participación de la familia.

- Diseñar intervenciones tempranas, orientadas a la disminución del riesgo de alteraciones físicas y psicológicas relacionadas con el trauma, mediante un cuidado individualizado a la persona, de acuerdo a sus necesidades, haciendo uso de estrategias de afrontamiento activo dirigidas a la resolución de problemas, el aprendizaje, la reevaluación positiva y las conductas orientadas a resolver los conflictos, ya que este tipo de afrontamiento generalmente resulta más adaptativo.

- Para próximos estudios, considerar las variables dolor e inmovilidad, que pueden afectar el proceso de afrontamiento y adaptación de las personas que experimentan traumas músculo esqueléticos.

- Realizar investigaciones de diseño cualitativo que permitan descubrir experiencias y vivencias de las personas que sufren este tipo de eventos traumáticos e inesperados.

- Asimismo resultaría interesante llevar a cabo investigaciones que permitan relacionar el proceso de afrontamiento y adaptación con el grado de responsabilidad de las personas en el accidente.

- Realizar estudios longitudinales que faciliten describir el proceso de afrontamiento y adaptación y su variación con respecto al tiempo.

\section{REFERENCIAS}

1. Quintero MT, Gómez M. El cuidado de Enfermería significa ayuda. Aquichan. 2010;Abril:8-18.

2. Richmond T AL. A model to advance nursing science in trauma practice and 
injury outcomes research. J Adv Nursing. 2011;Abril:2741-53.

3. Espinoza J. Atención básica y avanzada del politraumatizado. Acta médica peruana. Lima; 2011.

4. Vargas Castillo D. Comportamiento de muertes y lesiones por accidentes de transporte, Colombia [Internet]. Instituto Nacional de Medicina Legal y Ciencias Forenses-INMLCF Grupo Centro de Referencia Nacional sobre Violencia-GCRNV. 2014 [cited 2015 Dec 30]. Available from: http://www.medicinalegal.gov.co/documents/88730/1656998/Forensis+Interactivo+201 4.24-JULpdf.pdf/9085ad79-d2a9-4c0d-a17b-f845ab96534b

5. Martín Ayala JL OJ. Trastorno por estrés postraumático en víctimas de situaciones traumáticas. Psicothema. 2004;16 (1):45-9.

6. Cáceres Rodríguez DE, Montoya Camargo Z RHA. Intervención psicosocial para el incremento de la calidad de vida en pacientes con trauma craneoencefálico moderado a severo. Rev Colomb Psicol. 2003;12:60-72.

7. Zavala Yoe L, Rivas Lira RA APP. Validación del instrumento de estilos de enfrentamiento de Lazarus y Folkman en adultos de la ciudad de México. Rev Int Psicol y Educ. 2008;10 (2):159-182.

8. Vera B, Carbelo B VJ. La experiencia traumática desde la psicología positiva: resiliencia y crecimiento postraumático. Papeles del Psicólogo. 2006;27 (1):40-9.

9. Harms. L. After the accident: Survivors' perceptions of recovery following road trauma. Aust Soc Work. 57 (2):161-74.

10. Ching Hui W, Shiow Luan T EB. Post-traumatic stress disorder, depression, anxiety and quality of life in patients with traffic-related injuries. J Adv Nurs. 2005;52 (1):22-30.

11. Pérez Rincón E, González Forteza C, Ramos Lira M JTJ. TEPT en pacientes con lesiones no intencionales producidas en accidentes de tránsito. Salud Ment. 2007;30 (6):43-8.

12. Heron M, Kenardy J, Charlton E MY. A systematic review of predictors of posttraumatic stress disorder (PTSD) for adult road traffic crash survivors. Int. J. Care Inj. 2013;44:1413-1422.

13. Pirente N, Blum C, Wortberg S, Bostanci S, Berger E LR et al. Quality of life after multiple trauma: the effect of early onset psychotherapy on quality of life in trauma patients. Langenbecks Arch Surg. 2007;392:739-745.

14. Lee B, Chaboyer W WM. Predictors of Health-Related Quality of Life 3 Months after Traumatic Injury. J Nurs Scholarsh. 2008;40 (1):83-90.

15. Livneh H ME. The Impact of Perceptions of Health Control and Coping Modes on Negative Affect Among Individuals with Spinal Cord Injuries. J Clin Psychol Med Settings. 2011;18:243-256.

16. Paiva L, Aparecida LR, Silva Costa MC, Aparecida R DS. Experiencia del paciente politraumatizado y sus consecuencias. Rev Latino-Am Enfermagem. 2010;18 (6).

17. Mi-Ling Wong E, Wai-Chi Chan S CS. Effectiveness of an educational intervention on levels of pain, anxiety and self-efficacy for patients with musculoskeletal trauma. J Adv Nursing. 2010;66 (5).

18. O'Donnell M, Susannah Tipping WL, Holmes A, Rodney Judson S, Tracey Varker E, Richard A PE et al. Stepped early psychological intervention for posttraumatic stress disorder, other anxiety disorders, and depression following serious injury. J Trauma Stress. 2012;25(2):125-133.

19. Denholm C. Coping strategies during hospitalization and recovery following motor vehicle trauma: A personal account. Clin Psychol. 2009;13 (3):116-121.

20. Jeavons S, Horne DJ GK. Coping style and psychological trauma after road accidents. Psychol Health Med. 2000;5 (2). 
21. Rivka T, Freedman S, Bargai N, Boker R, Hadar H SA. Coping with Trauma: Narrative and Cognitive Perspectives Psychiatry. 2004;67(3):280-93.

22. Dorfel D, Rabe S KA. Coping strategies in daily life as protective and risk factors for post-traumatic stress in motor vehicle accident survivors. J Loss Trauma. 2008;13:422-440.

23. Rueda Ruiz MB, Aguado Díaz AL ARM. Estrés, afrontamiento y variables psicológicas intervinientes en el proceso de adaptación a la Lesión Medular (LM): una revisión de la bibliografía. Interv Psicosoc. 2008;17 (2):109-124.

24. Gustafsson M, Olof Persson L AA. A qualitative study of coping in the early stage of acute traumatic hand injury. J Clin Nurs. 2002;11:594-602.

25. Söderlund A LP. Whiplash-associated disorders - predicting disability from a process-oriented perspective of coping. Clin Rehabil. 2003;1 (7):101-107.

26. Hall P, Marshall J, Mercado A TG. Changes in Coping Style and Treatment Outcome Following Motor. Rehabil Psychol. 2011;56 (1):43-51.

27. Bernal C. Metodología de la investigación. Administración, economía, humanidades y ciencias sociales. $3^{a}$ edición. Hall; P, editor. Colombia; 119-120. p.

28. Sarmiento González P, Botero Giraldo J CCG. Validez de constructo de la Escala de Medición del Proceso de Afrontamiento y Adaptación de Roy, versión modificada en español. Index Enferm [Internet]. 2013;22(4):233-6. Available from: http://scielo.isciii.es/scielo.php?script=sci_arttext\&pid=S1132-

$12962013000300009 \&$ lng=es. http://dx.doi.org/10.4321/S1132-12962013000300009.

29. Gutiérrez López C, Veloza Gómez MM, Moreno Fergusson ME, Durán de Villalobos MM, López de Mesa C CO. Validez y confiabilidad de la versión en español del instrumento "Escala de medición del proceso de afrontamiento y adaptación de Callista Roy." Aquichán. 2007;7 (1):54-63.

30. Zavala S A- MJ. Ética e investigación. Rev Peru Med Exp y Salud Publica. 2011;28 (4):664.

31. Carrasco - Aldunate $P$, Rubio -Acuña M F-OD. Consentimiento informado: un pilar de la investigación clínica. Aquichán. 12 (1):32-41.

32. Salud M de. Resolución 008430 por la cual se establecen las normas académicas, técnicas y administrativas para la investigación en salud. Santa Fe de Bogotá; 1993.

33. Fitzharris M, Fildes B CJ. Anxiety, Acute- and Post-Traumatic Stress Symptoms Following Involvement in Traffic Crashes. 50th Annu Proc Assoc Adv Automot Med. 2006;

34. Sabana. CU de la. Campus 2.0: Noticias, Eventos y Opinión Incremento de 14 $\%$ de atención en Hospitalización. [Internet]. [cited 2015 Feb 9]. Available from: http://www.unisabana.edu.co/nc/la-sabana/campus 20/noticia/articulo/incremento-de14-de-atencion-en-hospitalizacion/

35. Walter R. Terapia cognitiva. Fundamentos teóricos y conceptualización del caso clínico. Grupo Edit. Bogotá; 2006. 177 p.

36. J. B. Asociación entre la capacidad del proceso de afrontamiento y adaptación y los grados de riesgo para la adherencia a los tratamientos farmacológicos y no farmacológicos en personas que presentan hipertensión arterial. Universidad Nacional de Colombia; 2011.

37. Callista R. Generating Middle Range Theory. From evidence to practice. Company SP, editor. New York; 2014.

38. J. V. El aprendizaje de los adultos. Afrontamiento cognitivo y adaptación de padres o madres con hijos en post operatorio de cardiopatía congénita en la Fundación Cardioinfantil de Bogotá. Universidad de la Sabana; 2010.

39. Reynoso Erazo L SI. Psicología y salud. Primera ed. Universidad Autónoma de México; editor. México; 2002. 75 p. 
40. Moos RH SJ. Coping resources and processes: Current concepts and measures. Citado por: Jiménez VF; Zapata LS; Díaz L. Capacidad de afrontamiento y adaptación de los familiares del paciente adulto hospitalizado en una unidad de cuidado intensivo. Aquichan. 2013;13 (2):159-72.

41. Gutiérrez C LC. Escala de Medición del proceso de afrontamiento y adaptación de Callista Roy: una propuesta metodológica para su interpretación. Rev Investig. 2009;6 (12):201-13.

42. Flórez Torres IE. Herrera Alarcón E. Carpio Jiménez E. Veccino Amador MA, Zambrano Barrios D RNY et al. Afrontamiento y adaptación en pacientes egresados de unidades de cuidado intensivo. Aquichán. 2011;11 (1):23-39.

43. Tan KL, Lim LM CL. Orthopedic patients' experience of motor vehicle accident in Singapore. J Compil Int Nurs Rev. 2008;55:110-116.

44. Hepp U, Moergeli S, Wittmann L SU. Coping with Serious Accidental Injury: A One-Year Follow-Up Study. Psychother Psychosom. 2005;74:379-386.

45. Pacella M, Irish L, Ostrowski S, Sledjeski E, Ciesla J FW et al. Avoidant Coping as a Mediator Between Peritraumatic Dissociation and Posttraumatic Stress Disorder Symptoms. J Trauma Stress. Vol. 24, N:317-325. 